\title{
Emergency percutaneous transluminal coronary angioplasty for intractable ventricular arrhythmias associated with acute anterior myocardial infarction
}

\author{
Adam P Fitzpatrick, Keith Dawkins, Neville Conway
}

\begin{abstract}
A previously fit marathon-running 54 year old man was admitted as an emergency having collapsed with chest pain caused by an acute transmural anterior myocardial infarction. He was initially resuscitated by his general practitioner then had recurrent episodes of ventricular flutter and fibrillation requiring continuing cardiopulmonary resuscitation and repeated defibrillation. During ambulance transfer and in the hospital emergency department he received appropriate intravenous antiarrhythmic drug treatment and a total of 63 transthoracic DC shocks, with good cardiac output between shocks. After his condition failed to stabilise in intensive care, an intra-aortic balloon pump was inserted and coronary angiography showed a proximal occlusion of the left anterior descending branch. Coronary angioplasty successfully re-opened the vessel with an excellent angiographic result. The intra-aortic balloon pump was withdrawn the following day and he was well enough to be discharged 7 days later. At 4 weeks he performed a satisfactory maximal exercise test and remains in New York Heart Association functional class $I$.
\end{abstract}

(Br Heart $\mathcal{f}$ 1993;69:453-454)

There is increasing interest in myocardial revascularisation in the setting of acute myocardial infarction in some subgroups of patients, such as those with cardiogenic shock, where evidence is accumulating in favour of acute intervention. ${ }^{1}$ Generally, acute intervention in myocardial infarction, even with adjuvant thrombolytic therapy, has been shown to offer no advantage over thrombolytic therapy alone. ${ }^{2}$ There are, however, no data available to guide acute intervention in the uncommon patient with intractable life-threatening arrhythmias as the primary complication of acute myocardial infarction. We report such a case with a favourable outcome.

Centre, Southampton General Hospital A P Fitzpatrick K Dawkins N Conway Correspondence to Dr A Fitzpatrick, Department of Electrophysiology, M312 Box 0214, Moffit Hospital, University of California, San Francisco, CA 94143
A 54 year old man collapsed with acute chest pain. He was previously extremely fit and well, and was a non-smoker and keen marathon runner. He was initially resuscitated by his family and his general practitioner who was promptly on the scene, and further by an experienced paramedical ambulance crew. He received approximately 20 DC defibrillations before arriving at the casualty department, where he was given appropriate doses of intravenous lignocaine, adrenaline, disopyramide, and flecainide via a central venous line at suitable intervals. He required a further series of DC defibrillations, and underwent endotracheal intubation and ventilation with correction of acid-base balance, without stabilisation of his rhythm, but with satisfactory cardiac output and tissue perfusion between episodes of arrhythmia. Twelve lead electrocardiography demonstrated the pattern of an acute transmural anterior myocardial infarction, with $\mathrm{q}$ wave formation in leads V1-V4 and ST segment elevation in V1-V6. In view of the failure to control the arrhythmias and the potentially arrhythmogenic effect of the drugs he had already received he was transferred from the intensive care unit to the cardiac catheterisation laboratory for coronary arteriography.

Intra-aortic balloon counter-pulsation (IABP) was quickly instituted and led to a clear reduction in the number and frequency of arrhythmic episodes. Without the need for repeated transthoracic defibrillation the patient became haemodynamically stable with a satisfactory blood pressure and peripheral perfusion and the return of urine output. Coronary angiography showed the right coronary artery to be dominant and normal. The left circumflex coronary artery was normal in appearance, and the left anterior descending (LAD) branch was occluded at its origin (fig 1A). Contrast ventriculography was not performed. An 0.014 inch intermediate angioplasty guidewire was successfully passed across the LAD occlusion which was then crossed with a $3.0 \mathrm{~mm}$ Alpha balloon dilatation catheter (all equipment from Advanced Cardiovascular Systems, Santa Clara, California, USA). After three inflations a satisfactory angiographic result was achieved (fig 1B). Later a cardiac radiological review of the angiographic appearances suggested occlusion rather than coronary spasm at the site of an atherosclerotic lesion. The patient's haemodynamic condition was stable and no further arrhythmias were noted. He remained on support by IABP for 12 hours, and was then weaned off counterpulsation which was withdrawn the following day. He remained free of ventricular arrhythmias and haemodynamically stable. He was mobilised gradually over the next 5 days and was discharged on 

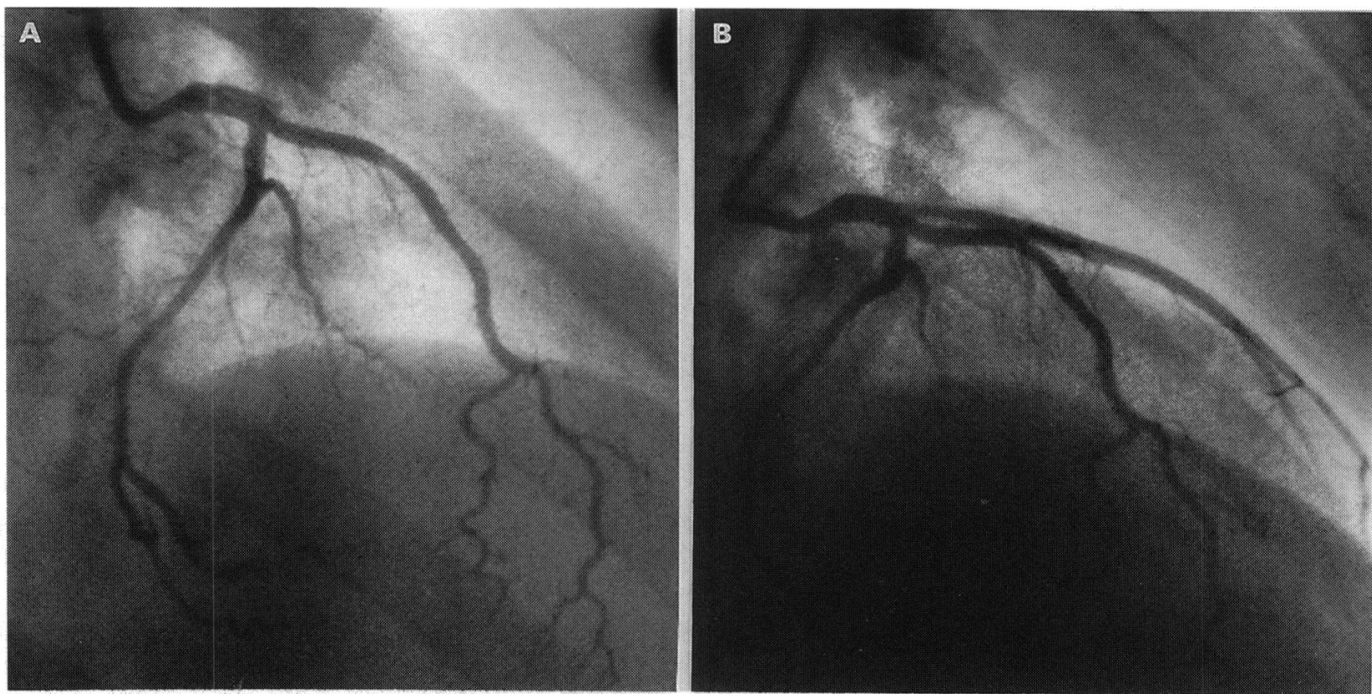

Figure 1 (A) Cineangiographic frame of a selective injection of the left coronary artery in right anterior oblique (RAO) projection showing proximal occlusion of the left anterior descending branch. (B) Post-angioplasty cineangiographic frame in $R A O$ projection showing wide patency of left anterior descending branch.

the seventh day after admission. He was treated with soluble aspirin $(150 \mathrm{mg}$ once a day and diltiazem ( $60 \mathrm{mg}$ three times a day) as was routine after PTCA in our unit. He performed an exercise test at a 4 week follow up, achieving 12 minutes of exercise on a modified Bruce protocol with satisfactory increases in heart rate and blood pressure and no evidence of myocardial ischaemia. $\mathrm{He}$ reported a good quality of life and no limitation of daily activities. He was advised to stop marathon running.

\section{Discussion}

This case illustrates that interventional coronary reperfusion in the setting of intractable ventricular arrhythmias complicating acute anterior myocardial infarction may be associated with an excellent outcome. Though coronary angioplasty with or without adjuvant thrombolytic therapy for all patients with acute myocardial infarction has not been shown to improve outcome, ${ }^{2}$ evidence is accumulating for a substantial benefit from acute intervention in certain subgroups of patients. One such group is patients with cardiogenic shock, for whom coronary reperfusion by angioplasty may considerably improve survival. Recent reports indicate that mortality, which in the past exceeded $80 \%,{ }^{4}$ may be reduced below $50 \%$ by intervention, with a persisting long-term benefit. ${ }^{13}$ Caution is needed, however, because randomised trials have not been done. In the Michigan series substantially more patients who underwent re-vascularisation for cardiogenic shock, and thereafter had a significant improvement in survival compared with those who were not re-vascularised, did so with haemodynamic support by IABP ( $84 \%)$. Such support was only given in $39 \%$ of those not re-vascularised by angioplasty, ${ }^{1}$ and these patients had a far worse outcome. This lack of randomisation, however, neglects confounding variables such as spontaneous thrombolysis and reperfusion of the infarct related vessel during clinical improvement that might accrue during IABP support. Randomised trials would remove remaining doubts. The start of IABP in our patient coincided with an improvement in his condition, and this may have been due to a reduction in left ventricular end diastolic pressure and therefore wall tension. Any improvement in coronary perfusion as a result of IABP seems unlikely, however, because the patient had a complete proximal occlusion of the left anterior descending coronary artery and otherwise unobstructed coronary arteries. Therefore the benefit noted in control of ventricular arrhythmias seems very likely to have been the result of the timely re-opening of the infarct-related vessel. In another series a fifth of patients undergoing emergency intervention for cardiogenic shock complicating acute myocardial infarction required active resuscitation during balloon angioplasty because of ventricular arrhythmias. In this series a greater than $50 \%$ overall survival, maintained at 2 year follow-up, affirms the value of intervention. $^{3}$

Re-opening of occluded coronary arteries during acute myocardial infarction may be associated with ventricular arrhythmias, and though none was evident here, this might be an important complication of percutaneous transluminal coronary angioplasty in other patients.

1 Moosvi A R, Khaja F, Villanueva L, Gheorghiade M, Douthat L, Goldstein S. Early revascularization improves survival in cardiogenic shock complicating acute myocardial infarction. $₹ \mathrm{Am}$ Coll Cardiol 1992;19:907-14.

2 Rogers W J, Baim D S, Gore J M, Brown B G, et al. Comparison of immediate invasive, delayed invasive, and conservative strategies after tissue-type plasminogen activator. Results of the Thrombolysis in Myocardial Infarction (TIMI) Phase II-A trial. Circulation 81:1457-76

3 Meyer P, Blanc P, Baudouy M, Morand P. Treatment of primary cardiogenic shock by coronary transluminal angioplasty during the acute phase of myocardial infarction. Arch Mal Coeur 1990;83:329-34.

4 Goldberg R J, Gore J M, Alpert J S, et al. Cardiogenic shock after acute myocardial infarction: incidence and mortality from a community-wide perspective 1975-1988. N Engl अ Med 1991;325:117-22. 